\title{
Recent Advances and Future Research Directions for Hearing Loss Treatment Based on Nanoparticles
}

\author{
Maximilian George Dindelegan $\mathbb{D}^{1,2}$ Cristina Blebea $\mathbb{D}^{2},{ }^{2}$ Maria Perde-Schrepler $\mathbb{D},^{3}$ \\ Anca Dana Buzoianu iD, 1 and Alma Aurelia Maniu (iD ${ }^{2}$ \\ ${ }^{1}$ Department of Clinical Pharmacology, "Iuliu Hatieganu" University of Medicine and Pharmacy, Cluj-Napoca, Romania \\ ${ }^{2}$ Department of Otorhinolaryngology, "Iuliu Hatieganu" University of Medicine and Pharmacy, Cluj-Napoca, Romania \\ ${ }^{3}$ Institute of Oncology "Prof. Dr. Ion Chiricuta", Cluj-Napoca, Romania
}

Correspondence should be addressed to Maximilian George Dindelegan; maximilian.dindelegan@gmail.com

Received 20 August 2021; Revised 11 November 2021; Accepted 21 December 2021; Published 17 January 2022

Academic Editor: Mohammad Owais

Copyright (c) 2022 Maximilian George Dindelegan et al. This is an open access article distributed under the Creative Commons Attribution License, which permits unrestricted use, distribution, and reproduction in any medium, provided the original work is properly cited.

\begin{abstract}
Sensorineural hearing loss is a prevailing health issue worldwide which usually occurs due to sensory inner ear cell destruction. These cells, once damaged, do not regenerate in mammals resulting in permanent hearing loss. Effective treatment of inner ear diseases is strongly correlated with efficient drug delivery to the inner ear. Researchers have made important efforts to deliver drugs locally to the inner ear in a safe and controllable manner. Different strategies and drug delivery systems were used to mitigate the resistance exerted by the multiple anatomical barriers found in the inner ear. Nanoparticles represent an option for sustained drug delivery to the inner ear. Nanocarrier-based systems can diffuse through the round and oval window membrane allowing for direct delivery into the inner ear components. Certain types of enhanced nanoparticles can focus on specific parts of the inner ear, providing targeted delivery which might represent the future therapy for sensorineural hearing loss. In this article, we will present the latest advances in the treatment of hearing loss based on nanoparticles.
\end{abstract}

\section{Introduction}

Sensorineural hearing loss (SNHL) is a common feature of many inner ear diseases, including presbycusis, genetic diseases, noise-induced hearing loss, ototoxic hearing loss, and autoimmune inner ear disease. Both environmental and genetic factors can alter hearing. Most of the elderly population and also 2-3 newborns out of 1000 suffer from impaired auditory function [1]. In Europe, presbycusis is the most common form of hearing impairment, affecting one in three people over the age of 65 , with the prevalence of the disease increasing with age. Hearing impairment leads to a considerable burden in terms of physical, social, and mental well-being, educational development, and employment [2].

SNHL develops due to damage to the cells of the inner ear as well as the disruption of the retrocochlear pathways from the spiral ganglion cells to the brain. The treatment of this condition poses a serious challenge on account of two main reasons: firstly, the sensory-neural transducer epithelial cells and nerve cells of the inner ear never recover once they have been destroyed, and thus, the loss of hearing is definitive. Secondly, the sensory-neural transducer epithelial cells are located in small and isolated inner ear compartments with protective barriers around them. Systemic delivery of substances to the cells of the inner ear is hindered by the presence of a blood-labyrinth barrier (BLB), which makes it difficult for active substances to exhibit their therapeutic effect at the level of the inner ear [3]. The only remaining option of partially restoring the auditory function is represented by hearing devices [4].

Recently, increasing knowledge of the pathophysiology of inner ear disorders allowed the development of different types of targeted local drug delivery methods [5]. Nanotechnology provides the potential of a noninvasive and targeted approach to deliver active molecules to specific structures 
within the inner ear for the treatment of SNHL. The delivery of biomaterials to targeted sites has remained challenging despite recent advances in the understanding of auditory neuronal survival biomolecular pathways [6].

The scope of this review is to present the latest advances in targeted drug delivery to the inner ear, which might lead to new therapeutic options for SNHL.

\section{Anatomical Obstacles of the Inner Ear in Drug Delivery}

The inner ear is a deep organ tightly encased in the temporal bone. The inner ear consists of two major compartments: the cochlea which contains the organ of Corti with the sensorineural hair cells for the auditory signal processing and the vestibule where balance is modulated. The bony otic capsule is a complex network of tubes having two compartments with membranous barriers filled with perilymph and endolymph. The bony otic capsule is the major anatomic and physiological barrier to inner ear drug delivery. Two structures separate the middle ear and the inner ear: the round window and the oval window [7].

The drugs that are delivered through intratympanic injection have to penetrate either the oval window membrane (OWM) or the round window membrane (RWM) [8]. The RWM represents the communication between the scala tympani of the cochlea and the middle ear. The membrane is composed of three different layers. The inner and outer layers of the RWM are made up of epithelial cells, while the middle layer is composed of connective tissue. The outer layer is the one that mostly modulates the permeability of the round window [9]. Because of this outer layer, the membrane permits the passage of a broad spectrum of substances. The semipermeable properties of the membrane pose another obstacle in the local delivery of substances into the inner ear. Different properties of the drugs, solubility, size, electrical properties, and concentration play also an important role in the efficiency of the diffusion. Another important factor to take into account is the decrease in permeability under certain local conditions, such as infection (otitis media), which alters the permeability of the RWM hindering the penetration of molecules into the cochlea [10-12]. The other access point into the inner ear is represented by the oval window. The molecules that pass through the oval window will arrive in the scala vestibuli of the inner ear. Compared to the round window, the OWM is partially covered by stapes' footplate. In spite of this more complex barrier, multiple studies have shown that molecules diffuse through the oval window during middle ear administration $[13,14]$.

In addition to the otic capsule, RWM and OWM, which represent obstacles in the diffusion of molecules into the perilymph of the inner ear, the eustachian tube (ET) also influences local drug delivery. Due to the communication between the middle ear and the nasopharynx through the ET, substances that are injected through the tympanic membrane are lost into the pharynx. Thus, sufficient therapeutical drug concentrations in the middle ear might not be achieved [15]. In order to avoid losing the fluid injected into the middle ear through the ET, patients are instructed to stay in a supine position with their head rotated towards the uninjected side for periods ranging from 10 to 30 minutes $[16,17]$.

\section{Drug Delivery to the Inner Ear: Administration Methods}

3.1. Systemic Delivery. There are different methods for achieving drug delivery to the inner ear. Systemic delivery of drugs is the current gold standard in drug delivery to the inner ear, but this type of drug delivery is hampered by the presence of the $\mathrm{BLB}$, which limits the diffusion of molecules into the inner ear [18]. Despite its easy administration, being noninvasive and widely used in current clinical treatment, the main limitations are poor targeting of the organs of interest, low local drug concentrations, uneven distribution, and important systemic side effects [19]. Because of the hindered diffusion of the molecules through the BLB, a higher plasmatic concentration of the active substance is required for therapeutic effect, which may lead to various side effects, especially from systemic corticosteroids, which are frequently used in the treatment of inner ear pathology $[3,20]$.

3.2. Local Delivery Methods. Besides systemic delivery, effective drug concentration can be achieved in the inner ear using local delivery of the therapeutic agent. Local delivery can be achieved either by intratympanic, intracochlear, or intracanalicular delivery. Each of these methods of delivery has particular advantages and disadvantages $[3,21]$.

3.2.1. Intratympanic Delivery. The advantage of the intratympanic delivery method is the possibility of delivering the substances either by direct injection through the tympanic membrane or through a catheter that is placed through the tympanic membrane for subsequent delivery of the drugs. Because of the local delivery of the molecules, the systemic effects of these molecules are avoided [22]. IT injection of a drug into the middle ear space allows for drug diffusion across the RWM and OWM into the inner ear bypassing the labyrinthine artery and BLB (Figure 1) [23].

The main disadvantage of intratympanic delivery is represented by the invasiveness of this method, the tympanic membrane being perforated in order to achieve the delivery of the drugs, with the risk of persistent tympanic membrane perforation and otitis media. Other disadvantages are represented by the clearance of the administered substances through the Eustachian tube and the subsequent resorption through the mucosa of the middle ear [24]. Different types of molecules can be administered using this route, the most widely used in clinical settings being corticosteroids for the treatment of sudden onset sensorineural hearing loss and Meniere's disease [25]. Various preclinical studies are attempting to use the intratympanic delivery method for extended delivery of corticosteroids using either hydrogels loaded with the active substance [26], microspheres loaded with corticosteroids [27], and nanoparticles used for prolonged drug delivery at the level of the inner ear [28].

3.2.2. Intracochlear Administration. This route of administration which also avoids the systemic effects of the drug is 


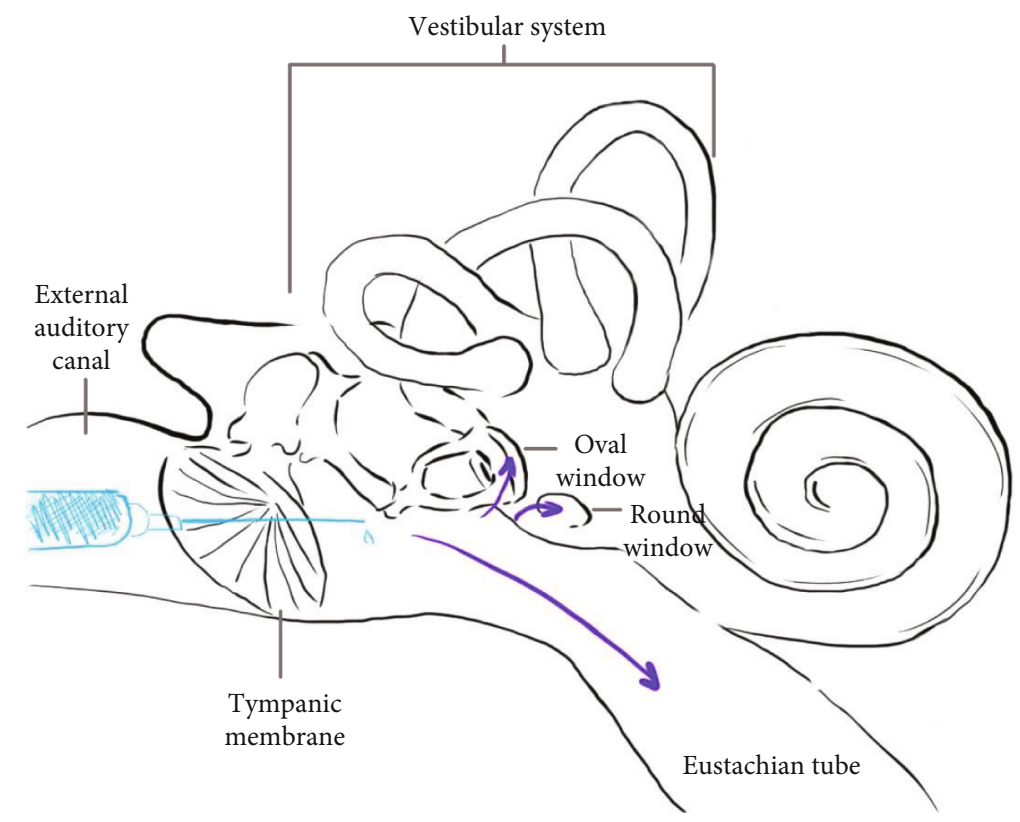

Figure 1: Intratympanic local drug delivery. Paths that can be taken by the substance injected into the middle ear.

not extensively used in the clinical settings. The primary limitation of this method is represented by the fact that surgical access to the cochlea is required, making the procedure a highly invasive one. However, the intracochlear route of administration is easily accessible when performing cochlear implants because the inner ear is already opened through the round window membrane [29].

Surgical trauma of the inner ear during cochlear implantation, caused by the insertion of an electrode in the inner ear, can also result in hearing impairment. Several types of substances are used for the treatment of these injuries, such as glucocorticoids, neurotrophins, and antiapoptotic substances [30-32].

3.2.3. Intracanalicular Drug Delivery. Intracanalicular drug delivery has so far been described only in preclinical settings, being a highly invasive procedure. The advantages of this method consist of the local delivery of the substance and direct access to the perilymph of the inner ear. Although this delivery site is not as extensively used in preclinical studies compared to the previously described sites, the local delivery of different substances at this level of the inner ear remains a viable option $[33,34]$.

\section{Current Treatment Options}

The current treatment options for sensorineural hearing loss caused by inner ear pathology are based on corticotherapy. The corticosteroids can be administered systemically or locally through the tympanic membrane [35]. There are multiple studies that show that systemic administration of corticosteroid drugs can treat different types of inner ear disease which lead to hearing loss, such as Meniere's disease, sudden onset hearing loss, hearing loss secondary to ototoxic drugs exposure, and other inner ear disorders [36, 37].
Because of the BLB, high doses of steroids are needed to reach therapeutic levels of drugs into the inner ear [3]. The high doses of corticosteroids can lead to various side effects such as osteoporosis, immunosuppression, hypertension, hyperglycemia, edema, gastrointestinal bleeding, poor wound healing, and increased risk of infection. Exposure for more than 14 days to systemic steroid treatment might lead to its most severe adverse effect which is represented by the hypothalamic-pituitary-adrenal axis inhibition [38].

The other route of administration for steroids is represented by local administration through intratympanic injection which does not lead to the systemic side effects which were mentioned before, but represents an invasive maneuver and necessitates repeated administration of the drug. Another drawback of the intratympanic corticosteroid injection is represented by the anatomical obstacles that were presented before which consist in the RWM, OWM, and the ET, all of them contributing to the impaired diffusion into the perilymph of the inner ear [15].

Nanoparticles have many advantages compared to the classical treatment with steroid drugs when treating sensorineural hearing loss. For example, one-single dose of dexamethasone encapsulated in polyethyleneglycol-coated polylactic acid (PEG-PLA) nanoparticles administered 1 hour before cisplatin treatment resulted in preservation of the functional and structural characteristics of the cochlea equivalent to the effect of 3 days of dexamethasone systemic injection in a guinea pig experimental model [39]. Dexamethasone-loaded nanoparticles were injected using the intratympanic route in a mouse experimental model, and their effect was compared with intratympanic dexamethasone injection for preventing ototoxic drug induced hearing loss. The nanoparticles loaded with dexamethasone provided improved hearing loss recovery in all tested hearing frequencies, and quantitative PCR showed that 
nanoparticles reduced the proinflammatory cytokines more than the standard dexamethasone treatment [28]. Nanoparticles also bring new treatment options for drug-induced hearing loss. In one study, two types of nanoparticles were synthesized. Polymeric nanoparticles loaded with dexamethasone and with $\alpha$-tocopheryl succinate were studied in vitro on HEI-OC1 cells both showing decreased caspase 3/7 activity, lower IL- $1 \beta$ release, and decreased cisplatin-induced toxicity. The same NP injected in the middle ear of Wistar rats showed protection against cisplatin-induced hearing loss compared to the control [40].

However, the expenditure of manufacturing and promoting NP for the clinical treatment of sensorineural hearing loss seems to be much higher than that of traditional therapy. This concern will most probably be overtaken in the future based on recent advances in manufacturing technology using 3D printing mechanism. In addition, producing NP with multiple functions will further reduce the NP cost therapy [41].

\section{Nanoparticle-Based Drug Delivery Systems (DDS)}

Nanoparticles play a major role in assisting local drug delivery to the inner ear. They are defined as solid particles with diameters smaller than $100 \mathrm{~nm}$ and molecular constructs of various organic or inorganic materials. Molecules can be added to the nanoparticles for functionalization, to aid in the delivery of substances, and to specifically target cells or organs, regulated by structural and signaling molecules. Customization of nanoparticles attributes can allow for noninvasive application, drug stabilization, controlled release, and surface modification for specific targeting [42, 43].

The use of nanoparticles allows noninvasive yet targeted drug delivery to the inner ear after systemic administration. With the advances in the field of nanoscale engineering, the concept of "smart" targeting is getting more and more applicability. The incorporation in the newly engineered nanostructures of receptor targeting antibodies, controlled release mechanisms, and characteristics to avoid premature elimination from the bloodstream may help to enhance their therapeutic effects and reduce off-target effects associated with systemic delivery [44].

The encapsulation of different types of drugs (small drugs, siRNA proteins, or peptides) within nanocarriers allows the increasing of their concentration and/or their residence time in the perilymph especially when the nanocarriers are dispersed within hydrogels. Nanocarriers migrate into the inner ear after specific targeting with or without losing their integrity [45].

NP-based DDS can improve the ability of drugs to cross the BLB giving hope for the treatment of hearing loss [46]. There are numerous systems for controlled drug delivery applicable for the different administration routes. Controlled drug release represents the control of time, amount, and location of release of the drug loaded into several types of delivery systems in order to improve the efficacy of the treatments.

The limited accessibility, small size, and high vulnerability of the inner ear limit the choices. This is the reason why such delivery systems should have high drug loading capacity and predictable release kinetics at the desired location in a desired time period, should be nontoxic, and should be eliminated shortly after the release of the active substance [47].

5.1. Lipid NP. Lipid NPs are used to deliver hydrophilic or lipophilic drugs, being highly biodegradable. They have been used as drug delivery systems to the inner ear [48].

5.1.1. Lipid Core Nanocapsules (LCN). Lipid core nanocapsules (LCN) contain a lipidic core made of triglycerides and mineral oils surrounded by stabilizing agents such as lecithin, polyethylene glycol (PEG), or poloxamere [21]. Their structure permits the inclusion of hydrophobic substances with different release patterns $[49,50]$. In a rat experimental model, $50 \mathrm{~nm}$ lipid core nanocapsules were able to pass through the RWM and enter the inner ear without inflicting any ototoxicity [51]. LCN loaded with dexamethasone were delivered to the inner ear through intratympanic injection. The amount of dexamethasone found in the inner ear perilymph was significantly higher compared to systemic dexamethasone administration [52]. LCN did not induce any toxicity in vivo in a mouse experimental model after 5 day systemic application of the nanocapsules [53].

5.1.2. Solid Lipid NP (SLNs). Solid lipid NP (SLNs) are submicron colloidal carriers that are capable of carrying high amounts of active substances making them a good option for improving drug performance $[48,54]$. They prove to be an alternative to liquid systems, because they form biocompatible lipids that become solidified at body temperature which in turn leads to an improved delivery of drugs [55]. SLNs were efficiently incorporated by HEI-OC1 cells without causing ototoxic effects. Low-dose hydrocortisone and dexametasone loaded into SLNs showed an increased survival time in HEI-OC1 cells treated with cisplatin compared to the standard treatment [48]. SLNs loaded with clozapine were used for the treatment of acute noise exposureinduced cochlea damage in an in vivo experimental model based on guinea pigs. The SLNs loaded with clozapine were able to exhibit protective effects on the cochlea. The hearing threshold shift in animals treated with SLNs loaded with clozapine were significantly lower compared to the untreated animals [56].

5.2. Phospholipid NP. NP based on a phospholipid structure have been extensively studied for inner ear drug delivery [57]. Yang et al. studied phospholipid nanoparticles that contained dexamethasone in a mouse experimental model for the prevention of ototoxicity induced by furosemide and kanamycin [28]. In their study, the authors delivered dexamethasone successfully into the inner ear hair cells using nanoparticles and obtained a significantly improved therapeutic result in preventing ototoxicity compared to the pure dexamethasone solution administered locally. In the auditory brainstem response (ABR) test, the particles provided improved hearing loss recovery compared to the Dex solution. Nanoparticles reduced the levels of proinflammatory cytokines, exhibiting superior anti-inflammatory effects than Dex solution. 


\subsection{Polymeric NP}

5.3.1. Polymersomes. Nanosized vesicles based on polymers called polymersomes can also be used for local delivery of substances into the cochlea. Roy et al. showed in their study that polymersomes functionalized with a certain peptide derived from the human nerve growth factor have a marked potential of binding solely to spiral ganglion neurons in cochlear explant cultures $[58,59]$. Zhang et al. studied polymersome with a Tet1 peptide sequence that binds specifically to a particular neuron receptor. The authors demonstrated that the nanoparticles targeted the spiral ganglion cells after intracochlear administration using a cochleostomy with subsequent administration of the particles directly into the perilymph, but they found no such polymersomes in the inner ear when they administered the particles through an intratympanic approach [60]. The fact that the nanoparticles were not able to pass through the RWM shows that the semipermeability of the membrane is capable of blocking even nanosized particles from reaching the perilymph. Polymersomes (PM) formed from synthetic amphiphilic block copolymers poly(ecaprolactone)-blockpoly(ethylene glycol) (PEG-b-PCL) were administered into five-month-old male Sprague Dawley rats either by topical RWM delivery using a gelatin sponge combined with an osmotic pump, intratympanic injection or through cochleostomy. The cochleostomy administration route found PEG-b-PCL PM in the mesothelial cells underneath the organ of Corti, spiral ligament, supporting cells in the organ of Corti, and spiral ganglion cells. The intratympanic injection method of delivering PM-induced diffusion of PM into the inner ear and the polymersomes could be found into the spiral ligament and the mesothelial cells beneath the organ of Corti. After the topical administration onto the RWM of PM, they could be found only into the spiral ligament. Regarding the vestibule of the inner ear, the best distribution was found after cochleostomy, followed by intratympanic injection and topical administration of the PM [61]. Another research group synthetized PEG-b-PCL $\mathrm{PM}$ and tested their diffusion into the inner ear and their potential as a drug delivery option. PM NPs labeled with fluorescent markers were applied onto the RWM of adult mice and were found after 24 hours in all the layers of the RWM, in the spiral ganglion of all cochlear turns and in a lower concentration in the organ of Corti and the lateral wall. The authors confirmed the PM NPs by performing hearing threshold monitoring and found no differences in the measurements made before the administration and 2 weeks after the administration of PM NPs. In order to test the potential of delivering therapeutic substances to the inner ear, PM were loaded with disulfiram, a neurotoxic agent. PM NPs loaded with disulfiram produced significant damage to the spiral ganglion as shown by histological assessment and also modified the hearing thresholds of the mice, inducing a significant hearing loss of $20-35 \mathrm{~dB}$ in the $8-25 \mathrm{kHz}$ range in the treated ear, with no hearing loss in the untreated ear [42].

5.3.2. PLGA NPs. Nanoparticles based on the polymer poly(lactic-co-glycolic acid) (PLGA) were also studied. Tetsuya et al. studied the distribution of nanoparticles loaded with rho- damine, a fluorescent substance, in the cochlea of guinea pigs. The authors found that after systemic delivery of rhodamineloaded nanoparticles, they did not pass through the BLB into the inner ear, but the local delivery of the PLGA nanoparticles to the RWM enabled the permeation of the particles through the RWM into the perilymph. [62]. Another study showed that PLGA nanoparticles loaded with dexamethasone applied onto the RWM led to a sustained concentration of dexamethasone in the perilymph of guinea pigs for up to 48 hours, offering a significant degree of protection against the ototoxicity of cisplatin [63]. Other than encapsulating and delivering a single drug, PLGA nanoparticles were shown to be able to deliver multiple substances at the same time. PLGA nanoparticles loaded with a combination of three drugs, notoginsenoside $\mathrm{R} 1$ (R1), ginsenoside Rg1 (Rg1), and ginsenoside Rb1 (Rb1), were synthetized. The three substances are extracted from Panax notoginseng and were shown to protect the spiral ganglion cells from cochlear ischemia $[64,65]$. The amount of R1, $\mathrm{Rg} 1$, and $\mathrm{Rb} 1$ in the perilymph of guinea pigs increased by $4.0-$ , 3.1-, and 7.1-fold when the substances were delivered encapsulated in nanoparticles compared to the delivery of the drugs in a free solution. This study demonstrates the ability of PLGA nanoparticles to carry multiple agents at the same time into the inner ear and increase their absorption $[65,66]$.

5.3.3. Chitosan NP. Chitosan is a natural polysaccharide polymer obtained from the deacetylation of chitin. It is a cationic polymer, considered nontoxic, biodegradable, and biocompatible $[67,68]$. It possess antifungal and antibacterial activity [69]. Several substances were encapsulated in chitosan particles to facilitate delivery such as insulin [70]. Chitosan-hydrogel-based nanoparticles also have a great potential of delivering therapeutics in a sustained manner into the inner ear without altering its structure [71]. Fluorescent traceable NPs were shown to pass into the vestibule of the inner ear through the OWM after intratympanic injection in a guinea pig experimental model [14]. As for all other nanostructures, the issue of toxicity is very important and represents an extensively studied field $[72,73]$.

5.4. Metallic Nanoparticles. Nanoparticles based on metallic substances represent another type of distinct nanoparticles studied in otology.

5.4.1. Silver Nanoparticles. Silver nanoparticles (Ag NPs) exhibit unique characteristics in the form of antiviral, antibacterial, and antifungal properties [74-77].

Because of their antibacterial properties, silver nanoparticles could be used for the treatment of chronic otitis media with resistant bacteria strains to antibiotic treatment and those cases where biofilm formation appears [78]. Chronic otitis media can lead to inner ear damage, which manifests as sensorineural hearing loss and vestibular pathology [79]. Zou et al. studied the distribution of AgNPs in the middle and inner ear after intratympanic injection using micro$\mathrm{CT}$, showing nanoparticles both in the middle ear and in a smaller concentration in the inner ear. The Ag NPs diffused through the OWM and RWM into the inner ear, demonstrating that the particles possess the necessary 
characteristics to pass through the semipermeable membranes of the ear in a dose-dependent manner. This information is relevant for designing clinical applications of $\mathrm{Ag}$ NPs in the treatment of chronic suppurative otitis media. The nanoparticle concentration and dosage must be thoroughly verified so it does not lead to adverse effects at the level of the inner ear [80].

5.4.2. Gold Nanoparticles. Gold nanoparticles are characterized by high chemical and physical stability. They were functionalized with biologically active organic molecules or atoms. Gold nanoparticles (AuNPs) were used for therapeutics and imaging applications and thus for the so-called theranostic purposes and showed excellent biocompatibility [81].

They were functionalized in through modification of the chemisorption and electrostatic interactions between drugs and AuNP in order to avoid drug degradation during endocytosis [82]. AuNP can be directly reduced and stabilized by chitosan (CS) to form a positively charged CS-AuNP core [83]. AuNPs can be further decorated with functional groups and ligands to target delivery to a specific site of interest. Some studies have proposed AuNP as a potential solution for inner ear theranostic applications [84].

The delivery of chitosan AuNP (CS-AuNP) was investigated and the potential enhancement using ultrasound microbubbles (USMBs). USMBs had an impressive efficiency in facilitating AuNP delivery to the inner ear probably by a transient disruption of the outer epithelium barrier and the tight junction- (TJ-) created paracellular barrier in the outer epithelium cells. They also showed that the administration of CS-AuNPs enhanced by USMBs in the tympanic cavity did not damage the integrity of the RWM nor did it affect the hearing thresholds [85].

5.4.3. Silica NP. Nanoporous silica nanoparticles (NPSNPs) with a diameter $<100 \mathrm{~nm}$ loaded with brain-derived neurotrophic factor (BDNF) were tested as long-term delivery systems for neurotrophins to spiral ganglion neurons (SGN) of the cochlea. In vitro experiments showed that SGN had a significantly higher survival rate in cell cultures that contained the BDNF-loaded NPs compared to the cell cultures with unloaded NPs. NPSNPs loaded with BDNF showed a long-term release pattern up to 39 days that increased the survival rate of SGNs [86].

5.4.4. Superparamagnetic Iron Oxide Nanoparticles (SPIONs). Superparamagnetic iron oxide nanoparticle (SPION) magnetic nanoparticles take advantage of the attraction generated by a magnetic force in order to pass through membranes. SPIONs are a promising option since their superparamagnetic properties allow them to be guided by magnetic forces to deliver the drug to the target with precision [87]. SPIONs are particularly interesting for drug delivery in central nervous system and inner ear since both structures are guarded by a blood-organ barrier, contain deep-seated, functionally sensitive, and fragile structures [88], and can benefit from targeted deliveries using an external magnetic field (EMF) $[89,90]$. Kopke et al. demonstrated that SPIONs could be pulled through an in vitro membrane model and in vivo guinea pig and rat RWM as well as fresh human cadaver RWM, in under 60 minutes with the help of a magnetic force of approximately 0.3 tesla [91].

Another study demonstrated that magnetic nanoparticles loaded with prednisolone sodium phosphate can migrate into the inner ear through the RWM with the help of a 0.5-tesla magnet and can unload their therapeutic load. An experimental mouse model which used cisplatin to induce ototoxicity was used. The authors found that the ears treated with prednisolone-loaded nanoparticles experienced substantially lower hearing loss compared to all other substances which were injected into the middle ear (saline, methylprednisolone, and unloaded magnetic nanoparticles) when tested with auditory brainstem response at $32 \mathrm{kHz}$. They also determined that hair cell preservation was more evident in mice treated with prednisolone-loaded nanoparticles compared to the other groups after cytocochleogram assessment [92].

In addition, the nanoparticles can be designed to include theranostic properties. Their surface-to-volume ratio can be significantly increased by the use of shells, and the number of ligands (targeting antibody or peptide), surface charge, hydrophobicity, and biocompatibility can also be modified [93]. The main application of SPIONs is in diagnostic imaging [94]. Although magnetic nanoparticles are an improvement over traditional intratympanic injections in animal models, these nanoparticles must be tested on humans as well. A concern remains in the fact that the distance between the magnet and the inner ear in humans is larger compared to the distance found in mice or other experimental models. Nonetheless, magnetic nanoparticles represent an interesting concept which was proven both in vitro and in vivo and could provide a solution for inner ear drug delivery in a clinical context.

5.5. Metal Oxide NP. There are also a number of metal-oxide nanoparticles that proved effective against infectious pathogens that can affect the ear.

Zirconium oxide nanoparticles possess strong inhibition against Escherichia coli and may possess inhibitory action against Staphylococcus aureus and Pseudomonas aeruginosa [95].

Calcium oxide nanoparticles possess bactericidal activity against Staphylococcus aureus and Escherichia coli [96].

Zinc oxide nanoparticles have been shown to have strong antimicrobial activity against Escherichia coli, Klebsiella pneumoniae, Pseudomonas aeruginosa, Staphylococcus aureus, and Enterococcus faecalis [97]. They were also shown to be efficient in the treatment of Candida albicans pathogenic yeast [98].

Copper oxide nanoparticles were shown to be effective in Candida albicans treatment [98].

\subsection{Advanced Nanocarrier Systems}

5.6.1. Cell Penetrating Peptides (CPP) Enhanced NP. Cellpenetrating peptides $(\mathrm{CPP})$ are short sequence $(<30)$ amino acid residues that have the ability to pass through the round window membrane $[58,99]$. The peptides can be attached to 
nanoparticles to enhance their diffusion through the RWM and OWM and to increase the cellular uptake of the nanoparticles. Yoon et al. developed nanoparticles based on the polyaminoacid (poly(2-hydroxyethyl-L-aspartamide)) (PHEA) and octadecylamine (C18) combined with the CPP oligoarginine peptide (Arg8). The authors found that the CPP-conjugated nanoparticles entered the HEI-OC1 cells at significant levels and that the PHEA-g-C18-Arg8 passed through the RWM of mice and led to increased Nile red staining and green fluorescent protein (GFP) expression in different parts of the inner ear [100]. The authors went on and studied the same PHEA-g-C18-Arg8 (PCA) nanoparticles loaded with dexamethasone (Dex) to enhance the gene delivery potential of the nanoparticles. The Dex-PCA-gene nanoparticles established an anti-inflammatory effect and enhanced gene expression in mouse HEI-OC1 cells when tested in vitro [101]. Another study that used CPPenhanced nanoparticles chose SS-31 peptide which targets mitochondrial cardiolipin [102]. In another study, SS-31 peptide-conjugated geranylgeranylacetona (GGA) was loaded into PLGA nanoparticles. GGA represents a heat shock protein inducer that was delivered to the mitochondria of hair cells with the help of the SS-31 peptide. The authors found that SS-31-GGA-loaded PLGA nanoparticles were effective at preventing gentamicin-induced hair cell death in an experimental zebrafish model [103].

5.6.2. Nanoparticle-Hydrogel Systems. Drug clearance through the Eustachian tube represents a particular issue, as previously stated. Different types of hydrogels were synthesized to avoid drug loss from the middle ear. The hydrogels that have been used are thermosensitive. They react to the increased temperature of the middle ear and transform from a liquid state into solid state, preventing the active substance from leaking out of the middle ear through the Eustachian tube. Poloxamer 407 solution is such a hydrogel, in which the molecules assemble into micelles that undergo a gelification process when the temperature increases [104]. Poloxamer 407 used as a dexamethasone drug delivery vehicle led to significant dexamethasone levels in the perilymph of the inner ear for at least 10 days after the hydrogel was inserted into the middle ear, with minimal systemic exposure. On the other hand, a single intratympanic injection of dexamethasone led to detectable levels of dexamethasone in the perilymph for a maximum of $24 \mathrm{~h}$, but with an increased decline in concentration after a few hours [105].

In addition to being used as drug carriers, hydrogels have also been used as carriers for nanoparticles. Such a drug delivery system made of PLGA nanoparticles containing interferon $\alpha$-2b loaded into a thermosensitive hydrogel based on chitosan and glycerol phosphate was shown to aid controllable drug release. This system provided detectable interferon levels in the cochlea of guinea pigs for as long as 72 hours, providing a 3 -fold increase in retention time compared to the interferon solution injected through an intratympanic method, which was detectable for 24 hours. Nanoparticles loaded with interferon alone and hydrogel loaded with interferon were found to provide a prolonged delivery with detectable interferon levels up to 48 hours.

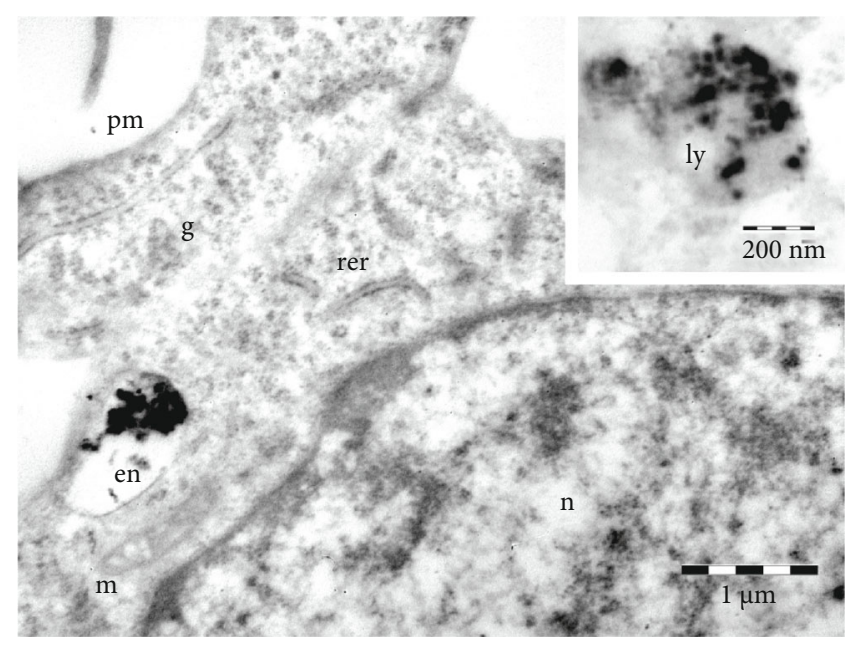

FIgURE 2: Ultrastructure of a HEI-OC1 cell incubated for $24 \mathrm{~h}$ with AgNPs with a diameter of $110 \mu \mathrm{m}$. The NPs are present in an endosome (en) and in a lysosome (ly) (inset). G: glycogen granules; m: mitochondrion; pm: plasma membrane; rer: rough endoplasmic reticulum.

The combination enhanced their effects and provided nanoparticle-assisted drug release with greater efficacy [106].

Another drug delivery system based on nanoparticles and hydrogels is represented by a chitosan-glycerophosphate thermosensitive hydrogel and liposomal nanoparticles. The release pattern of this nanohydrogel was studied both in vitro and in vivo. The in vitro studies showed that the nanoparticles maintained their stability for more than two weeks in physiological conditions (release rate $<1 \% /$ day). The nanohydrogel was able to release unmodified liposomes in a controlled manner. In a mouse mode, 24 hours after the application of the hydrogel loaded with nanoparticles on the round window membrane, the mice were euthanatized. Intact liposomes were identified in the Corti's organ with the help of fluorescent microscopy. [71].

\subsection{Active Targeting of Nanocarriers}

5.7.1. PEGylated Polymersomes Coated with Tet1 Peptide. The nanoparticles were also decorated with indocarbocyanine dye. After transtympanic injection, both targeted and untargeted polymersomes were found in the RWM, spiral ligament, scala vestibuli and tympani, and Reissner's membrane with no accumulation in the cochlear nerve [60].

5.7.2. PEGylated PLA Nanoparticles Coated with A666 Peptide. Coumarin-6 was used as tracer, and the NP contained also dexamethasone. $2 \mathrm{~h}$ after RWM application, coumarin- 6 was found in the perinuclear region of outer hair cells and in modiolus, to a greater extent for targeted nanoparticles than for untargeted ones. Dexamethasone releases in perilymph, quantified by LC-MS, were not significantly different between A666-covered and untargeted PEGylated PLA nanoparticles [107].

5.7.3. Chitosan Gel Containing PEGylated Liposomes Coated with Prestin-Targeting Peptide-1. The NPs contained plasmid encoding TdTomato and as a tracer carboxyfluorescein, 
rhodamine or cyanine 5-labeled lipid. After RWM application for 2-5 days, $5 \%$ of the targeted liposomes was found in the perilymph at day 2 . Both targeted and untargeted liposomes were found in the outer hair cells of mid and basal turns of cochlea. Targeted liposomes reached the apical turn unlike untargeted ones. Both TdTomato and carboxyfluorescein were expressed not only by outer hair cells but also in other tissues [108].

\section{Nanoparticle Safety}

The safety of these nanoparticles and their toxicity represents an actively studied field. The steps needed to obtain end-product nanoparticles deal with different limitations ranging from cost ineffectiveness to the formation of toxic products and the use of hazardous chemicals [109]. In order to avoid the potential toxicities of the nanoparticles, safety studies must be conducted on a broad concentration range of nanocarriers, because the nanocarrier concentration should be adjusted to obtain efficient doses of the drug in the sites of interest [45]. Several studies conducted on different formulations of nanoparticles found a lack of toxic effects such as modifications of the hearing thresholds for Streptavidin-covered SPIONs [89], for Hyaluronic acid gel containing PEGylated liposomes [110]. No histological damages were observed in the structures of the inner ear after treatments with A665peptide-covered AuNPs in hydrogel [84] or PLGA NP mixed with protamine [111].

Other studies, mostly regarding chitosan nanocarriers, found slight inflammatory changes in the cochlea but no long-term hearing loss $[112,113]$.

AgNPs represent one of the most used and studied type of nanoparticle up to date. Besides the medical uses, AgNPs are being used in the food industry, personal care, and in household items [114]. These types of nanoparticles were found to be extensively present in wastewater from the whole European territory leading to concerns regarding their environmental effects and toxicity potential $[115,116]$. The toxicity of AgNPs is proportional with particles' size. The toxicity of silver nanoparticles is mainly caused by oxidative stress which can lead to mithocondrial dysfunction and increase of the permeability of the cellular membrane [117-119]. The most cytotoxic AgNPs tested in vitro on the HEI-OC1 and HaCaT (spontaneously immortalized human keratinocyte cell line) cells were represented by the smallest nanoparticles $(5 \mathrm{~nm})$, followed by the $25 \mathrm{~nm}$ NPs, the $50 \mathrm{~nm}$ NPs, and the $110 \mathrm{~nm}$ NPs (Figure 2) [119].

Zou et al. attempted to evaluate the toxicity of AgNPs at the level of the external, middle, and inner ear after transtympanic injection in rats and also their effect in the $\mathrm{BALB} / \mathrm{c}$ 3T3 cell line. The authors detected apoptosis in the cochlea after exposure to different concentrations of AgNPs. The destruction of inner ear cells was greater in the vestibule compared to the cochlea especially in the $200 \mu \mathrm{g} / \mathrm{ml} \mathrm{AgNP}$ solution. Exposure to the lower concentration AgNPs showed that the strial marginal cells were the most sensitive cells to the nanoparticle toxicity, followed by the osteocytes from the cochlear shell, osseous spiral limbus, and epithelial cells of Reissner's membrane. The cochlear hair cells were among the least affected. Higher concentrations led to universal cell death in the vestibule and in the cochlea, in the portion situated between the basal part of the cochlea and the second lower turn. [120].

The possible toxicity induced by magnetic nanoparticles (MNP) was studied not only in otology but also in other medical fields, the MNP exerting nervous system, cardiovascular, respiratory, immune, urinary system, and liver toxicity $[121,122]$. Inhaled MNPs with sizes lower than $50 \mathrm{~nm}$ were shown to reach a variety of organs as well, ranging from testes, brain, liver, and spleen to lungs. A lower concentration of MNPs was distributed to the heart, nasal cavity, kidney, and ovaries. They could pass through both the blood-brain barrier and blood-testes barrier but no apparent toxicity could be observed in their study [123].

There are multiple factors that determine the biodegradation and toxicity of nanoparticles. One should not take into account just the components from which nanoparticles are made, but as multiple data show the size of nanoparticles represents one of the most important aspects regarding their toxicity. Even if there are studies that show that some NPs have ototoxicity in an experimental model, there is not any clear report on the ototoxicity of different nanomaterials in humans [124]. The toxic effects of each type of nanoparticles should be comprehensively studied before their use in clinical settings both in an in vitro and in vivo experimental models [121]. Summary of nanoparticle types and their effects are shown in Table 1.

\section{Conclusions and Future Directions}

Sensorineural hearing loss represents an important and difficult to treat health problem.

Currently, the administration of therapeutics via intratympanic or intracochlear injections has proven to be more effective than systemic medications. Even in these circumstances, local drug delivery to the inner ear is hindered by the inner ear barriers. In recent years, there has been a growing interest in the synthesis and study of nanoparticles as targeted drug delivery systems to different parts of the inner ear. Various types of nanoparticles have been investigated for the treatment of inner ear pathology, many of them offering multiple benefits over the classical administration methods by offering great stability and specificity, a higher capacity of drug storing, and the feasibility of targeted drug delivery. The nanoparticles have some distinct advantages compared to other options. Some of the nanoparticles are able to diffuse through the RWM and OWM and target specific components of the inner ear, depending on their specific properties.

Recently, there is a clear trend towards the development of advanced nanocarrier systems such as nanocarriers incorporated in hydrogels, nanoparticles with cell-penetrating peptides, and nanoparticles functionalized with a targeting ligand increasing the permeability of the round window membrane. Nanocarriers appear to be globally safe for the inner ear. The long-term toxicity needs to be evaluated for side effects due to nanocarrier degradation. In the future, cross-sectional studies evaluating nanocarrier 


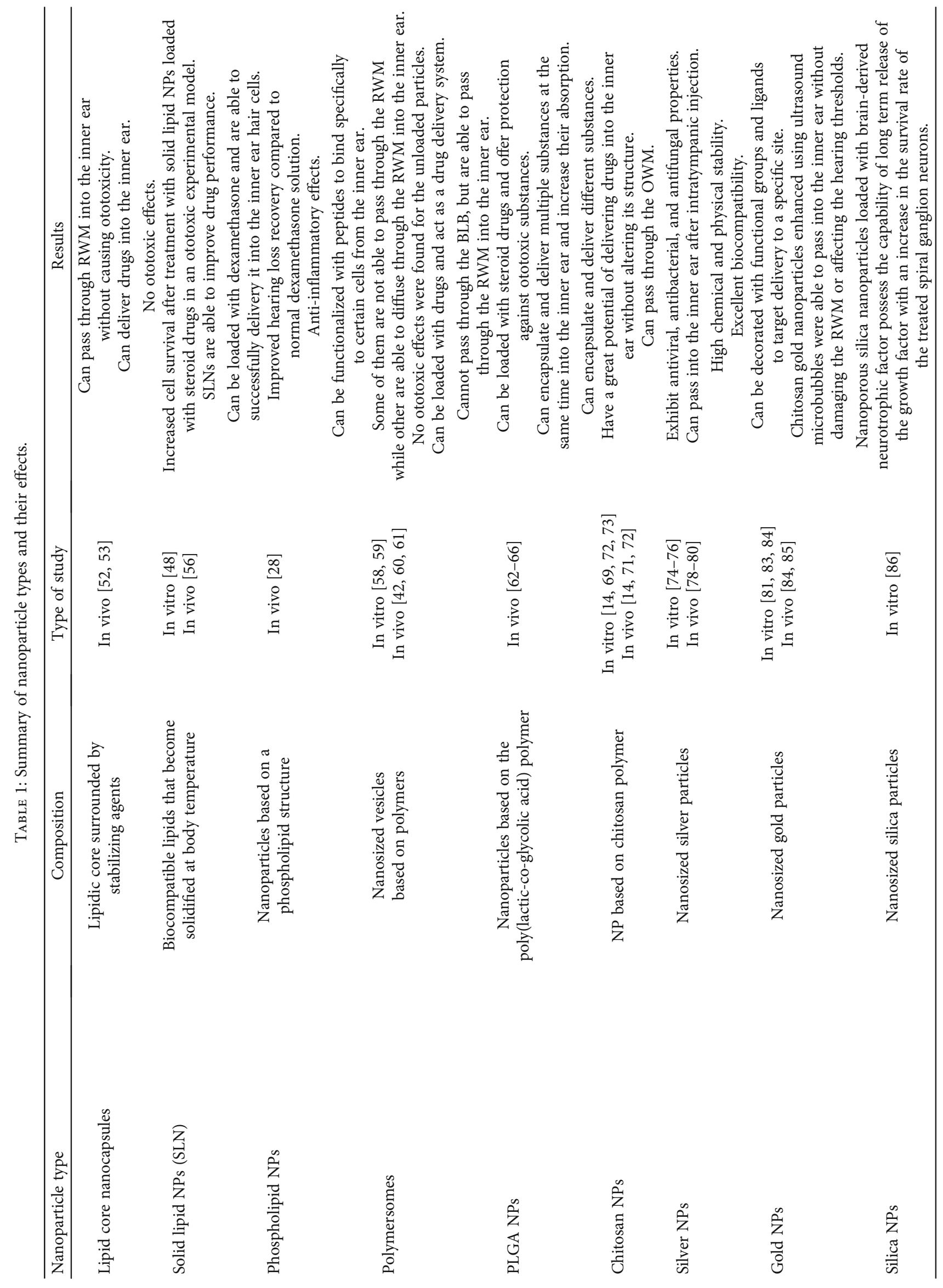




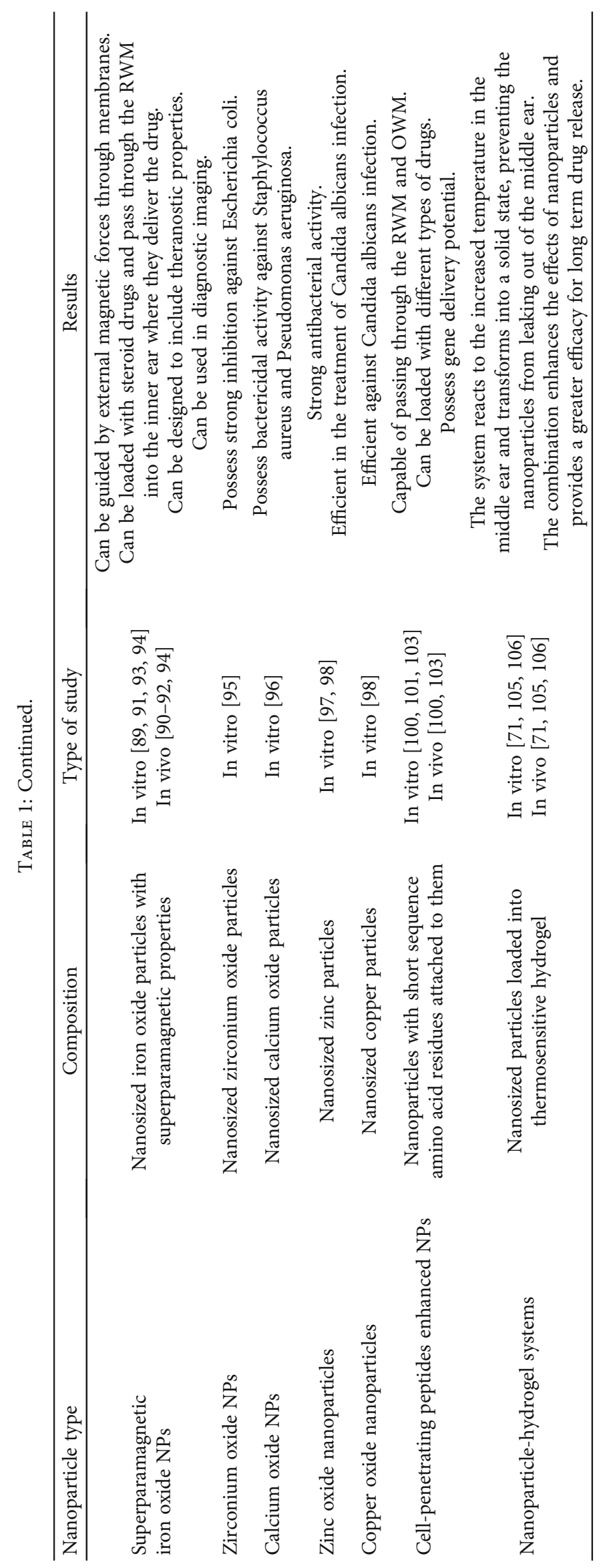


biodistribution, drug delivery, safety, and efficacy must be developed. In addition, the issues related to the manufacturing of nanomaterials and cost problems are hoped to be solved by $3 \mathrm{D}$ printing technology.

To conclude, with all the concerns regarding the efficiency and safety of nanoparticles, the outlook for the application of this technology in clinical settings is optimistic. Further studies in this field, combined with the positive results that have already been achieved, point to an important place of nanotechnology in the treatment of inner ear disease.

\section{Data Availability}

No data were used to support this study.

\section{Conflicts of Interest}

The authors declare that they have no conflicts of interest.

\section{Acknowledgments}

This work was supported by The Executive Agency for Higher Education, Research, Development, and Innovation Funding, Romania (project number PN-III-P2-2.1-PED2019-3813).

\section{References}

[1] B. Vohr, "Overview: infants and children with hearing loss?part I," Mental Retardation and Developmental Disabilities Research Reviews, vol. 9, no. 2, pp. 62-64, 2003.

[2] N. Quaranta, F. Coppola, M. Casulli et al., "Epidemiology of age related hearing loss: a review," Hearing, Balance and Communication, vol. 13, no. 2, pp. 77-81, 2015.

[3] S. Nyberg, N. J. Abbott, X. Shi, P. S. Steyger, and A. Dabdoub, "Delivery of therapeutics to the inner ear: the challenge of the blood-labyrinth barrier," Science Translational Medicine, vol. 11, no. 482, pp. 1-12, 2019.

[4] B. D. Cavaleriu, D. Mârțu V., L. Hrițcu, O. Manolache R., and L. Rădulescu M., "Idiopathic sudden hearing loss: oxidative status before and after corticoid treatment," Archives of Biological Sciences, vol. 67, no. 4, pp. 1297-1302, 2015.

[5] E. Colombo, P. Feyen, M. R. Antognazza, G. Lanzani, and F. Benfenati, "Nanoparticles: a challenging vehicle for neural stimulation," Frontiers in neuroscience, vol. 10, 2016.

[6] S. G. Kujawa and M. C. Liberman, "Adding insult to injury: cochlear nerve degeneration after 'temporary' noise-induced hearing loss," The Journal of Neuroscience, vol. 29, pp. 14077-14085, 2009.

[7] F. Piu and K. M. Bishop, "Local drug delivery for the treatment of neurotology disorders," Frontiers in Cellular Neuroscience, vol. 13, pp. 1-11, 2019.

[8] A. A. Mikulec, S. K. Plontke, J. J. Hartsock, and A. N. Salt, "Entry of substances into perilymph through the bone of the otic capsule after intratympanic applications in guinea Pigs," Otology \& Neurotology, vol. 30, no. 2, pp. 131-138, 2009.

[9] Y. C. Lin, H. C. Chen, H. K. Chen et al., "Ultrastructural changes associated with the enhanced permeability of the round window membrane mediated by ultrasound microbubbles," Frontiers in Pharmacology, vol. 10, pp. 1-13, 2020.
[10] M. V. Goycoolea, D. Muchow, and P. Schachern, "Experimental studies on round window structure: function and permeability," Laryngoscope, vol. 98, pp. 1-20, 1988.

[11] M. V. Goycoolea, "Clinical aspects of round window membrane permeability under normal and pathological conditions," Acta Oto-Laryngologica, vol. 121, pp. 437-447, 2001.

[12] K. Ikeda and T. Morizono, "Changes of the permeability of round window membrane in otitis media," Archives of Otolaryngology-Head \& Neck Surgery, vol. 114, pp. 895-897, 1988.

[13] E. B. King, A. N. Salt, G. E. Kel, H. T. Eastwood, and S. J. O'Leary, "Gentamicin administration on the stapes footplate causes greater hearing loss and vestibulotoxicity than round window administration in guinea pigs," Hearing Research, vol. 304, pp. 159-166, 2013.

[14] S. Ding, S. Xie, W. Chen et al., "Is oval window transport a royal gate for nanoparticle delivery to vestibule in the inner ear?," European Journal of Pharmaceutical Sciences, vol. 126, pp. 11-22, 2019.

[15] A. N. Salt and K. Hirose, "Communication pathways to and from the inner ear and their contributions to drug delivery," Hearing Research, vol. 362, pp. 25-37, 2018.

[16] O. J. Ungar, O. Handzel, L. Haviv et al., "Optimal head position following intratympanic injections of steroids, as determined by virtual reality," Otolaryngology-Head and Neck Surgery, vol. 161, no. 6, pp. 1012-1017, 2019.

[17] S. H. Park, C. Park, J. Y. Seo, J. H. Cho, and I. S. Moon, "How long should patients remain in the supine treatment position after intratympanic dexamethasone injection?," Laryngoscope, vol. 124, no. 12, pp. 2807-2810, 2014.

[18] E. C. Bielefeld and M. J. Kobel, "Advances and challenges in pharmaceutical therapies to prevent and repair cochlear injuries from noise," Frontiers in Cellular Neuroscience, vol. 13, pp. 1-13, 2019.

[19] C. L. Nieman, N. S. Reed, and F. R. Lin, "Otolaryngology for the internist: hearing loss," The Medical Clinics of North America, vol. 102, pp. 977-992, 2018.

[20] B. Szeto, H. Chiang, C. Valentini, M. Yu, J. W. Kysar, and A. K. Lalwani, "Inner ear delivery: challenges and opportunities," Laryngoscope Investigative Otolaryngology, vol. 5, no. 1, pp. 122-131, 2020.

[21] N. el Kechai, F. Agnely, E. Mamelle, Y. Nguyen, E. Ferrary, and A. Bochot, "Recent advances in local drug delivery to the inner ear," International Journal of Pharmaceutics, vol. 494, no. 1, pp. 83-101, 2015.

[22] R. Suryanarayanan, V. R. Srinivasan, and G. O'Sullivan, "Transtympanic gentamicin treatment using Silverstein MicroWick in Mnire's disease patients: long term outcome," The Journal of Laryngology and Otology, vol. 123, pp. 45-49, 2009.

[23] L. Li, T. Chao, J. Brant, B. O'Malley Jr., A. Tsourkas, and D. Li, "Advances in nano-based inner ear delivery systems for the treatment of sensorineural hearing loss," Advanced Drug Delivery Reviews, vol. 108, pp. 2-12, 2017.

[24] A. N. Salt and S. K. R. Plontke, "Local inner-ear drug delivery and pharmacokinetics," Drug Discovery Today, vol. 10, pp. 1299-1306, 2005.

[25] D. M. Barrs, J. S. Keyser, C. Stallworth, and J. T. McElveen Jr., "Intratympanic steroid injections for intractable M??ni??re???s disease," Laryngoscope, vol. 111, no. 12, pp. 2100-2104, 2001. 
[26] A. N. Salt, J. Hartsock, S. Plontke, C. LeBel, and F. Piu, "Distribution of dexamethasone and preservation of inner ear function following intratympanic delivery of a gel-based formulation," Audiology and Neurotology, vol. 16, no. 5, pp. 323-335, 2011.

[27] N. H. Dormer, J. Nelson-Brantley, H. Staecker, and C. J. Berkland, "Evaluation of a transtympanic delivery system in _Mus musculus_for extended release steroids," European Journal of Pharmaceutical Sciences, vol. 126, pp. 3-10, 2019.

[28] K. J. Yang, J. Son, S. Y. Jung et al., "Optimized phospholipidbased nanoparticles for inner ear drug delivery and therapy," Biomaterials, vol. 171, pp. 133-143, 2018.

[29] S. K. Plontke, G. Götze, T. Rahne, and A. Liebau, "Intracochlear drug delivery in combination with cochlear implants," $H N O$, vol. 65, no. S1, pp. 19-28, 2017.

[30] C. Jolly, C. Garnham, H. Mirzadeh et al., "Electrode features for hearing preservation and drug delivery strategies," Cochlear Implants and Hearing Preservation, vol. 67, pp. 28-42, 2009.

[31] D. Rejali, V. A. Lee, K. A. Abrashkin, N. Humayun, D. L. Swiderski, and Y. Raphael, "Cochlear implants and ex vivo BDNF gene therapy protect spiral ganglion neurons," Hearing Research, vol. 228, no. 1-2, pp. 180-187, 2007.

[32] A. A. Eshraghi and T. R. Van De Water, "Cochlear implantation trauma and noise-induced hearing loss: apoptosis and therapeutic strategies," The Anatomical Record Part A: Discoveries in Molecular, Cellular, and Evolutionary Biology: An Official Publication of the American Association of Anatomists, vol. 288, pp. 473-481, 2006.

[33] V. Raghu, Y. Ramakrishna, R. F. Burkard, and S. G. Sadeghi, "A novel intracochlear injection method for rapid drug delivery to vestibular end organs," Journal of Neuroscience Methods, vol. 341, article 108689, 2020.

[34] J. Husseman and Y. Raphael, "Gene therapy in the inner ear using adenovirus vectors," Advances in Oto-Rhino-Laryngology, vol. 66, pp. 37-51, 2009.

[35] P. Salvador, F. Moreira, and R. Fonseca, "Idiopathic sudden sensorineural hearing loss: effectiveness of salvage treatment with low-dose intratympanic dexamethasone," The Journal of Otolaryngology, vol. 16, pp. 6-11, 2021.

[36] J. Li and L. Ding, "Effectiveness of steroid treatment for sudden sensorineural hearing loss: a meta-analysis of randomized controlled trials," The Annals of Pharmacotherapy, vol. 54, pp. 949-957, 2020.

[37] Y. Liu, Q. Chen, and Y. Xu, "Research progress in refractory sudden hearing loss: steroid therapy," Journal of International Medical Research, vol. 48, 2020.

[38] D. M. Williams, "Clinical pharmacology of corticosteroids," Respiratory Care, vol. 63, pp. 655-670, 2018.

[39] C. Sun, X. Wang, D. Chen, X. Lin, D. Yu, and H. Wu, "Dexamethasone loaded nanoparticles exert protective effects against Cisplatin- induced hearing loss by systemic administration," Neuroscience Letters, vol. 619, pp. 142148, 2016.

[40] S. Martín-Saldaña, R. Palao-Suay, M. R. Aguilar, R. RamírezCamacho, and J. San Román, "Polymeric nanoparticles loaded with dexamethasone or $\alpha$-tocopheryl succinate to prevent cisplatin-induced ototoxicity," Acta Biomaterialia, vol. 53, pp. 199-210, 2017.

[41] Y. Z. Zhang, Y. Wang, Q. Jiang, J. K. el-Demellawi, H. Kim, and H. N. Alshareef, "MXene printing and patterned coating for device applications," Advanced Materials, vol. 32, no. 21, p. 1908486, 2020.

[42] D. Buckiová, S. Ranjan, T. A. Newman et al., "Minimally invasive drug delivery to the cochlea through application of nanoparticles to the round window membrane," Nanomedicine, vol. 7, no. 9, pp. 1339-1354, 2012.

[43] I. Pyykkö, J. Zou, A. Schrott-Fischer, R. Glueckert, and P. Kinnunen, "An overview of nanoparticle based delivery for treatment of inner ear disorders," in Sokolowski B. (eds) Auditory and Vestibular Research. Methods in Molecular Biology, Humana Press, New York, NY, 2016.

[44] S. Mura, J. Nicolas, and P. Couvreur, "Stimuli-responsive nanocarriers for drug delivery," Nature Materials, vol. 12, pp. 991-1003, 2013.

[45] C. Jaudoin, F. Agnely, Y. Nguyen, E. Ferrary, and A. Bochot, "Nanocarriers for drug delivery to the inner ear: physicochemical key parameters, biodistribution, safety and efficacy," International Journal of Pharmaceutics, vol. 592, article 120038, 2021.

[46] X. An and D. Zha, "Development of nanoparticle drugdelivery systems for the inner ear," Nanomedicine, vol. 15, pp. 1981-1993, 2020.

[47] K. Mäder, E. Lehner, A. Liebau, and S. K. Plontke, "Controlled drug release to the inner ear: concepts, materials, mechanisms, and performance," Hearing Research, vol. 368, pp. 49-66, 2018.

[48] B. Cervantes, L. Arana, S. Murillo-Cuesta, M. Bruno, I. Alkorta, and I. Varela-Nieto, "Solid lipid nanoparticles loaded with glucocorticoids protect auditory cells from cisplatin-induced ototoxicity," Journal of Clinical Medicine, vol. 8, no. 9, p. 1464, 2019.

[49] F. Valente, L. Astolfi, E. Simoni et al., "Nanoparticle drug delivery systems for inner ear therapy: an overview," Journal of Drug Delivery Science and Technology, vol. 39, pp. 28-35, 2017.

[50] E. Jäger, C. G. Venturini, F. S. Poletto et al., "Sustained release from lipid-core nanocapsules by varying the core viscosity and the particle surface area," Journal of Biomedical Nanotechnology, vol. 5, no. 1, pp. 130-140, 2009.

[51] Y. Zhang, W. Zhang, M. Löbler et al., "Inner ear biocompatibility of lipid nanocapsules after round window membrane application," International Journal of Pharmaceutics, vol. 404, no. 1-2, pp. 211-219, 2011.

[52] G. Chen, S. X. Hou, P. Hu, Q. H. Hu, D. D. Guo, and Y. Xiao, "In vitro dexamethasone release from nanoparticles and its pharmacokinetics in the inner ear after administration of the drug-loaded nanoparticles via the round window," Journal of Southern Medical University, vol. 28, pp. 1022-1024, 2008.

[53] J. Hureaux, F. Lagarce, F. Gagnadoux et al., "Toxicological study and efficacy of blank and paclitaxel-loaded lipid nanocapsules after i.v. administration in mice," Pharmaceutical Research, vol. 27, no. 3, pp. 421-430, 2010.

[54] V. Mishra, K. K. Bansal, A. Verma et al., "Solid lipid nanoparticles: emerging colloidal nano drug delivery systems," Pharmaceutics, vol. 10, no. 4, p. 191, 2018.

[55] S. Agarwal, R. S. R. Murthy, S. L. Harikumar, and R. Garg, "Quality by design approach for development and characterisation of solid lipid nanoparticles of quetiapine fumarate," Current Computer-Aided Drug Design, vol. 16, no. 1, pp. 73-91, 2020. 
[56] N. Wang, X. Gao, M. Li, Y. Li, and M. Sun, "Use of solid lipid nanoparticles for the treatment of acute acoustic stressinduced cochlea damage," Journal of Nanoscience and Nanotechnology, vol. 20, no. 12, pp. 7412-7418, 2020.

[57] G. Bozzuto and A. Molinari, "Liposomes as nanomedical devices," International Journal of Nanomedicine, vol. 10, pp. 975-999, 2015.

[58] S. Roy, A. H. Johnston, T. A. Newman et al., "Cell-specific targeting in the mouse inner ear using nanoparticles conjugated with a neurotrophin-derived peptide ligand: potential tool for drug delivery," International Journal of Pharmaceutics, vol. 390, no. 2, pp. 214-224, 2010.

[59] L. Messager, J. Gaitzsch, L. Chierico, and G. Battaglia, "Novel aspects of encapsulation and delivery using polymersomes," Current Opinion in Pharmacology, vol. 18, pp. 104-111, 2014.

[60] Y. Zhang, W. Zhang, A. H. Johnston, Newman, and I. Pyykko, "Targeted delivery of Tet1 peptide functionalized polymersomes to the rat cochlear nerve," International Journal of Nanomedicine, vol. 7, pp. 1015-1022, 2012.

[61] Y. Zhang, W. Zhang, A. H. Johnston, T. A. Newman, I. Pyykkö, and J. Zou, "Comparison of the distribution pattern of PEG-b-PCL polymersomes delivered into the rat inner ear via different methods," Acta Oto-Laryngologica, vol. 131, no. 12, pp. 1249-1256, 2011.

[62] T. Tamura, T. Kita, T. Nakagawa et al., "Drug delivery to the cochlea using PLGA nanoparticles," Laryngoscope, vol. 115, no. 11, pp. 2000-2005, 2005.

[63] C. Sun, X. Wang, Z. Zheng et al., "A single dose of dexamethasone encapsulated in polyethylene glycol-coated polylactic acid nanoparticles attenuates cisplatin-induced hearing loss following round window membrane administration," International Journal of Nanomedicine, vol. 10, pp. 3567-3579, 2015.

[64] K. Fujita, N. Hakuba, R. Hata et al., "Ginsenoside Rb1 protects against damage to the spiral ganglion cells after cochlear ischemia," Neuroscience Letters, vol. 415, no. 2, pp. 113-117, 2007.

[65] H. Cai, X. Wen, L. Wen et al., "Enhanced local bioavailability of single or compound drugs delivery to the inner ear through application of plga nanoparticles via round window administration," International Journal of Nanomedicine, vol. 9, pp. 5591-5601, 2014.

[66] C. Rathnam, S. D. Chueng, Y. M. Ying, K. B. Lee, and K. Kwan, "Developments in bio-inspired nanomaterials for therapeutic delivery to treat hearing loss," Frontiers in Cellular Neuroscience, vol. 13, pp. 1-13, 2019.

[67] A. Ali and S. Ahmed, "A review on chitosan and its nanocomposites in drug delivery," International Journal of Biological Macromolecules, vol. 109, pp. 273-286, 2018.

[68] J. Sharifi-Rad, C. Quispe, M. Butnariu et al., "Chitosan nanoparticles as a promising tool in nanomedicine with particular emphasis on oncological treatment," Cancer Cell International, vol. 21, no. 1, pp. 1-21, 2021.

[69] T. M. Tawfik and A. M. A. El-Masry, "Preparation of chitosan nanoparticles and its utilization as novel powerful enhancer for both dyeing properties and antimicrobial activity of cotton fabrics," Biointerface Research in Applied Chemistry, vol. 11, pp. 13652-13666, 2021.

[70] K. Al Rubeaan, M. Rafiullah, and S. Jayavanth, "Oral insulin delivery systems using chitosan-based formulation: a review," Expert Opinion on Drug Delivery, vol. 13, pp. 223-237, 2016.
[71] S. A. Lajud, D. A. Nagda, P. Qiao et al., "A novel chitosanhydrogel-based nanoparticle delivery system for local inner ear application," Otology \& Neurotology, vol. 36, no. 2, pp. 341-347, 2015.

[72] S. Jesus, A. P. Marques, A. Duarte et al., "Chitosan nanoparticles: shedding light on immunotoxicity and hemocompatibility," Frontiers in bioengineering and biotechnology, vol. 8, 2020.

[73] R. M. Saeed, I. Dmour, and M. O. Taha, "Stable chitosanbased nanoparticles using polyphosphoric acid or hexametaphosphate for tandem ionotropic/covalent crosslinking and subsequent investigation as novel vehicles for drug delivery," Frontiers in Bioengineering and Biotechnology, vol. 8, pp. 121, 2020.

[74] M. A. Radzig, V. A. Nadtochenko, O. A. Koksharova, J. Kiwi, V. A. Lipasova, and I. A. Khmel, "Antibacterial effects of silver nanoparticles on gram-negative bacteria: influence on the growth and biofilms formation, mechanisms of action," Colloids and Surfaces. B, Biointerfaces, vol. 102, pp. 300306, 2013.

[75] D. Xiang, Y. Zheng, Y. Zheng et al., "Inhibition of A/Human/ Hubei/3/2005 (H3N2) influenza virus infection by silver nanoparticles in vitro and in vivo," International Journal of Nanomedicine, vol. 8, pp. 4103-4114, 2013.

[76] R. J. B. Pinto, A. Almeida, S. C. M. Fernandes et al., "Antifungal activity of transparent nanocomposite thin films of pullulan and silver against_Aspergillus niger_," Colloids and Surfaces. B, Biointerfaces, vol. 103, pp. 143-148, 2013.

[77] D. C. Gheorghe, A. G. Niculescu, A. C. Bîrcă, and A. M. Grumezescu, "Nanoparticles for the treatment of inner ear infections," Nanomaterials, vol. 11, no. 5, p. 1311, 2021.

[78] X. Gu, Y. Keyoumu, L. Long, and H. Zhang, "Detection of bacterial biofilms in different types of chronic otitis media," European Archives of Oto-Rhino-Laryngology, vol. 271, no. 11, pp. 2877-2883, 2014.

[79] C. W. Chang, P. W. Cheng, and Y. H. Young, "Inner ear deficits after chronic otitis media," European Archives of OtoRhino-Laryngology, vol. 271, no. 8, pp. 2165-2170, 2014.

[80] J. Zou, M. Hannula, S. Misra et al., "Micro CT visualization of silver nanoparticles in the middle and inner ear of rat and transportation pathway after transtympanic injection," Journal of nanobiotechnology, vol. 13, no. 1, pp. 5-9, 2015.

[81] D. Pissuwan, C. Gazzana, S. Mongkolsuk, and M. B. Cortie, "Single and multiple detections of foodborne pathogens by gold nanoparticle assays," Wiley Interdisciplinary Reviews: Nanomedicine and Nanobiotechnology, vol. 12, pp. 1-19, 2020.

[82] C. A. Hong and Y. S. Nam, "Functional nanostructures for effective delivery of small interfering RNA therapeutics," Theranostics, vol. 4, pp. 1211-1232, 2014.

[83] S. Guo, Y. Huang, Q. Jiang et al., "Enhanced gene delivery and siRNA silencing by gold nanoparticles coated with chargereversal polyelectrolyte," ACS Nano, vol. 4, no. 9, pp. 5505$5511,2010$.

[84] M. N. Kayyali, L. Brake, A. J. Ramsey, A. C. Wright, B. W. O’Malley, and D. D. Li, "A novel nano-approach for targeted inner ear imaging," Journal of nanomedicine \& nanotechnology, vol. 8, pp. 4-9, 2017.

[85] Y. C. Lin, C. P. Shih, H. C. Chen et al., "Ultrasound microbubble-facilitated inner ear delivery of gold nanoparticles involves transient disruption of the tight junction barrier in 
the round window membrane," Frontiers in Pharmacology, vol. 12, pp. 1-17, 2021.

[86] N. Schmidt, J. Schulze, D. P. Warwas et al., "Long-term delivery of brain-derived neurotrophic factor (BDNF) from nanoporous silica nanoparticles improves the survival of spiral ganglion neurons in vitro," PLoS One, vol. 13, no. 3, pp. 123, 2018.

[87] C. Guigou, A. Lalande, N. Millot, K. Belharet, and A. Bozorg Grayeli, "Use of super paramagnetic iron oxide nanoparticles as drug carriers in brain and ear: state of the art and challenges," Brain Sciences, vol. 11, no. 3, p. 358, 2021.

[88] R. Mittal, S. A. Pena, A. Zhu et al., "Nanoparticle-based drug delivery in the inner ear: current challenges, limitations and opportunities," Artificial cells, nanomedicine, and biotechnology, vol. 47, no. 1, pp. 1312-1320, 2019.

[89] Y. Nguyen, C. Celerier, R. Pszczolinski et al., "Superparamagnetic nanoparticles as vectors for inner ear treatments: driving and toxicity evaluation," Acta oto-laryngologica, vol. 136, no. 4, pp. 402-408, 2016.

[90] G. Leterme, C. Guigou, A. Oudot et al., "Superparamagnetic nanoparticle delivery to the cochlea through round window by external magnetic field: feasibility and toxicity," Surgical Innovation, vol. 26, no. 6, pp. 646-655, 2019.

[91] R. D. Kopke, R. A. Wassel, F. Mondalek et al., "Magnetic nanoparticles: inner ear targeted molecule delivery and middle ear implant," Audiology and Neurotology, vol. 11, no. 2, pp. 123-133, 2006.

[92] B. Ramaswamy, S. Roy, A. B. Apolo, B. Shapiro, and D. A. Depireux, "Magnetic nanoparticle mediated steroid delivery mitigates cisplatin induced hearing loss," Frontiers in cellular neuroscience, vol. 11, 2017.

[93] S. Liu, B. Yu, S. Wang, Y. Shen, and H. Cong, "Preparation, surface functionalization and application of $\mathrm{Fe}_{3} \mathrm{O}_{4}$ magnetic nanoparticles," Advances in Colloid and Interface Science, vol. 281, article 102165, 2020.

[94] M. Shevtsov, B. Nikolaev, Y. Marchenko et al., "Targeting experimental orthotopic glioblastoma with chitosan-based superparamagnetic iron oxide nanoparticles (CS-DXSPIONs)," International Journal of Nanomedicine, vol. Volume 13, pp. 1471-1482, 2018.

[95] J. B. Fathima, A. Pugazhendhi, and R. Venis, "Synthesis and characterization of $\mathrm{ZrO} 2$ nanoparticles-antimicrobial activity and their prospective role in dental care," Microbial Pathogenesis, vol. 110, pp. 245-251, 2017.

[96] S. M. Dizaj, F. Lotfipour, M. Barzegar-Jalali, M. H. Zarrintan, and K. Adibkia, "Antimicrobial activity of the metals and metal oxide nanoparticles," Materials Science and Engineering: $C$, vol. 44, pp. 278-284, 2014.

[97] P. M. Narayanan, W. S. Wilson, A. T. Abraham, and M. Sevanan, "Synthesis, characterization, and antimicrobial activity of zinc oxide nanoparticles against human pathogens," Bionanoscience, vol. 2, no. 4, pp. 329-335, 2012.

[98] A. Karimiyan, H. Najafzadeh, M. Ghorbanpour, and S. H. Hekmati-Moghaddam, "Antifungal effect of magnesium oxide, zinc oxide, silicon oxide and copper oxide nanoparticles against Candida albicans," Zahedan Journal of Research in Medical Sciences, vol. 17, no. 10, pp. 25-27, 2015.

[99] Y. Maitani and Y. Hattori, "Oligoarginine-PEG-lipid particles for gene delivery," Expert Opinion on Drug Delivery, vol. 6, pp. 1065-1077, 2009.
[100] J. Y. Yoon, K. J. Yang, D. E. Kim et al., "Intratympanic delivery of oligoarginine-conjugated nanoparticles as a gene (or drug) carrier to the inner ear," Biomaterials, vol. 73, pp. 243-253, 2015.

[101] J. Y. Yoon, K. J. Yang, S. N. Park, D. K. Kim, and J. D. Kim, "The effect of dexamethasone/cell-penetrating peptide nanoparticles on gene delivery for inner ear therapy," International Journal of Nanomedicine, vol. Volume 11, pp. 61236134, 2016.

[102] H. H. Szeto, "First-in-class cardiolipin-protective compound as a therapeutic agent to restore mitochondrial bioenergetics," British Journal of Pharmacology, vol. 171, pp. 20292050, 2014.

[103] X. Kuang, S. Zhou, W. Guo, Z. Wang, Y. Sun, and H. Liu, "Ss31 peptide enables mitochondrial targeting drug delivery: a promising therapeutic alteration to prevent hair cell damage from aminoglycosides," Drug Delivery, vol. 24, no. 1, pp. 1750-1761, 2017.

[104] G. Dumortier, J. L. Grossiord, F. Agnely, and J. C. Chaumeil, "A review of poloxamer 407 pharmaceutical and pharmacological characteristics," Pharmaceutical Research, vol. 23, no. 12, pp. 2709-2728, 2006.

[105] X. Wang, L. Dellamary, R. Fernandez et al., "Dose-dependent sustained release of dexamethasone in inner ear cochlear fluids using a novel local delivery approach," Audiology and Neurotology, vol. 14, no. 6, pp. 393-401, 2009.

[106] J. Dai, W. Long, Z. Liang, L. Wen, F. Yang, and G. Chen, “A novel vehicle for local protein delivery to the inner ear: injectable and biodegradable thermosensitive hydrogel loaded with PLGA nanoparticles," Drug Development and Industrial Pharmacy, vol. 44, no. 1, pp. 89-98, 2018.

[107] X. Wang, Y. Chen, Y. Tao, Y. Gao, D. Yu, and H. Wu, “A666conjugated nanoparticles target prestin of outer hair cells preventing cisplatin-induced hearing loss," International Journal of Nanomedicine, vol. Volume 13, pp. 7517-7531, 2018.

[108] M. Kayyali, J. Wooltorton, A. Ramsey et al., "A novel nanoparticle delivery system for targeted therapy of noiseinduced hearing loss," Journal of Controlled Release, vol. 279, pp. 243-250, 2018.

[109] A. Kahru and K. Savolainen, "Potential hazard of nanoparticles: from properties to biological and environmental effects," Toxicology, vol. 269, pp. 89-91, 2010.

[110] N. el Kechai, E. Mamelle, Y. Nguyen et al., "Hyaluronic acid liposomal gel sustains delivery of a corticoid to the inner ear," Journal of Controlled Release, vol. 226, pp. 248-257, 2016.

[111] H. Cai, Z. Liang, W. Huang, L. Wen, and G. Chen, "Engineering PLGA nano-based systems through understanding the influence of nanoparticle properties and cell-penetrating peptides for cochlear drug delivery," International Journal of Pharmaceutics, vol. 532, no. 1, pp. 55-65, 2017.

[112] M. Shimoji, B. Ramaswamy, M. I. Shukoor et al., “Toxicology study for magnetic injection of prednisolone into the rat cochlea," European Journal of Pharmaceutical Sciences, vol. 126, pp. 33-48, 2019.

[113] J. F. Lafond, M. Shimoji, B. Ramaswamy et al., "Middle ear histopathology following magnetic delivery to the cochlea of prednisolone-loaded iron oxide nanoparticles in rats," Toxicologic Pathology, vol. 46, no. 1, pp. 101-106, 2018.

[114] C. Völker, C. Boedicker, J. Daubenthaler, M. Oetken, and J. Oehlmann, "Comparative toxicity assessment of nanosilver 
on three daphnia species in acute, chronic and multigeneration experiments," PLoS One, vol. 8, no. 10, 2013.

[115] E. Dumont, A. C. Johnson, V. D. J. Keller, and R. J. Williams, "Nano silver and nano zinc-oxide in surface waters - Exposure estimation for Europe at high spatial and temporal resolution," Environmental Pollution, vol. 196, pp. 341-349, 2015.

[116] C. O. Hendren, X. Mesnard, J. Dröge, and M. R. Wiesner, "Estimating production data for five engineered nanomaterials as a basis for exposure assessment," Environmental Science \& Technology, vol. 45, no. 7, pp. 2562-2569, 2011.

[117] L. Zapór, "Effects of silver nanoparticles of different sizes on cytotoxicity and oxygen metabolism disorders in both reproductive and respiratory system cells," Archives of Environmental Protection, vol. 42, no. 4, pp. 32-47, 2016.

[118] T. J. Webster, D. Gorth, and D. Rand, "Silver nanoparticle toxicity in drosophila: size does matter," International Journal of Nanomedicine, vol. 343, 2011.

[119] M. Perde-Schrepler, A. Florea, I. Brie et al., "Size-dependent cytotoxicity and genotoxicity of silver nanoparticles in cochlear cells in vitro," Journal of Nanomaterials, vol. 2019, 12 pages, 2019.

[120] J. Zou, H. Feng, M. Mannerström, T. Heinonen, and I. Pyykkö, "Toxicity of silver nanoparticle in rat ear and BALB/c 3T3 cell line," Journal of nanobiotechnology, vol. 12, no. 1, pp. 1-10, 2014.

[121] J. Kim, J. Shin, and M. Cho, "Magnetic nanoparticles: an update of application for drug delivery and possible toxic effects," Archives of Toxicology, vol. 86, pp. 685-700, 2012.

[122] J. S. Kim, T. Yoon, K. N. Yu et al., "Toxicity and tissue distribution of magnetic nanoparticles in mice," Toxicological Sciences, vol. 89, no. 1, pp. 338-347, 2006.

[123] J. Kwon, S. Hwang, H. Jin et al., "Body distribution of inhaled fluorescent magnetic nanoparticles in the mice," Journal of Occupational Health, vol. 50, no. 1, pp. 1-6, 2008.

[124] S. Murugadoss, I. V. Vrček, B. Pem et al., "A strategy towards the generation of testable adverse outcome pathways for nanomaterials," ALTEX-Alternatives to animal experimentation, vol. 38, no. 4, pp. 580-594, 2021. 